\title{
Síndrome de la cimitarra
}

\author{
Eva Buller Viqueira, Gema Soler Cifuentes y David Soler Cifuentes
}

Centro de Salud de Barbate. Barbate. Cádiz (España).

\section{Correspondencia:}

Eva Buller Viqueira. Centro de Salud de Barbate. Avda. del Mar, s/n. C.P. 11160. Barbate (Cádiz). España.

Correo electrónico: miji_77@ yahoo.com.

Recibido el 27 de marzo de 2015.

Aceptado para su publicación el 28 de abril de 2015 .

\section{RESUMEN}

El síndrome de la cimitarra consiste en un retorno venoso pulmonar anómalo. En esta patología la vena pulmonar conecta con la vena cava inferior en lugar de la aurícula izquierda. Presentamos el caso de un hombre de 40 años con el diagnóstico reciente de síndrome de la cimitarra. El paciente estaba asintomático desde el punto de vista cardiológico. Tras el cauteloso estudio de cardiología y varias pruebas complementarias, entre ellas ecografías, resonancia nuclear magnética, TAC y radiología de tórax se le diagnosticó la enfermedad.

PALABRAS CLAVE: Síndrome de Cimitarra. Dextrocardia. Cardiopatías Congénitas.

\section{ABSTRACT}

\section{Scimitar syndrome}

Scimitar syndrome consists in an anomalous pulmonary venous return. In this pathology the pulmonary vein connects to the inferior vena cava instead of the left atrium. We present the case of a 40-year-old man with scimitar syndrome. The patient had no cardiological clinical symptoms. After a cautious cardiological study and various complementary tests -among them ecography, magnetic resonance imaging, computed tomography and chest $\mathrm{X}$-ray- the diagnosis was achieved.

KEY WORDS: Scimitar Syndrome. Dextrocardia. Heart Defects, Congenital.

\section{INTRODUCCIÓN}

El Síndrome de la cimitarra o síndrome de drenaje venoso anómalo del pulmón derecho fue descrito por primera vez en 1936 por Cooper. Consiste en un drenaje anómalo de la vena pulmonar derecha a la vena cava inferior o a la aurícula derecha por encima de la desembocadura de la vena cava inferior. También se ha descrito dextrocardia, hipoplasia pulmonar derecha y otras malformaciones cardiacas y pulmonares. Este drenaje venoso anómalo es visible en una radiografía simple de tórax como una opacidad con densidad agua, lineal curva, paralelo al borde derecho cardiaco hasta el ángulo cardiofrénico; la imagen se asemeja a un sable turco llamado cimitarra y que da el nombre al síndrome y al signo radiográfico (acuñado por Neill en 1966) ${ }^{1-3}$.

\section{CASO CLÍNICO}

Presentamos el caso de un varón de 40 años sin antecedentes personales de interés y sin hábitos tóxicos. Acude a la consulta de atención primaria por catarro y de forma casual se detecta un soplo sistólico, por lo que se solicita un electrocardiograma (ECG), objetivándose extrasístoles ventriculares aisladas. Se deriva a cardiología para completar el estudio.

Asintomático desde el punto de vista cardiológico. Los cardiólogos solicitan entre otras pruebas complementarias radiografía de tórax (figura 1), eco- 
cardiograma transtorácico y transesofágico, resonancia nuclear magnética (RNM) cardiaca, Holter y tomografía axial computarizada (TAC) cardiaca, llegando finalmente al diagnóstico de síndrome de la cimitarra (drenaje venoso pulmonar anómalo parcial) con persistencia de la vena cava superior izquierda, comunicación interauricular tipo seno coronario y disfunción sistólica ligera de ventrículo izquierdo. Tratan al paciente con ramipril $2,5 \mathrm{mg} /$ $24 \mathrm{~h}$, bisoprolol 2,5 mg / $24 \mathrm{~h}$, ácido acetilsalicílico $100 \mathrm{mg} / 24 \mathrm{~h}$ y plantean la posibilidad de corrección quirúrgica de la anomalía.

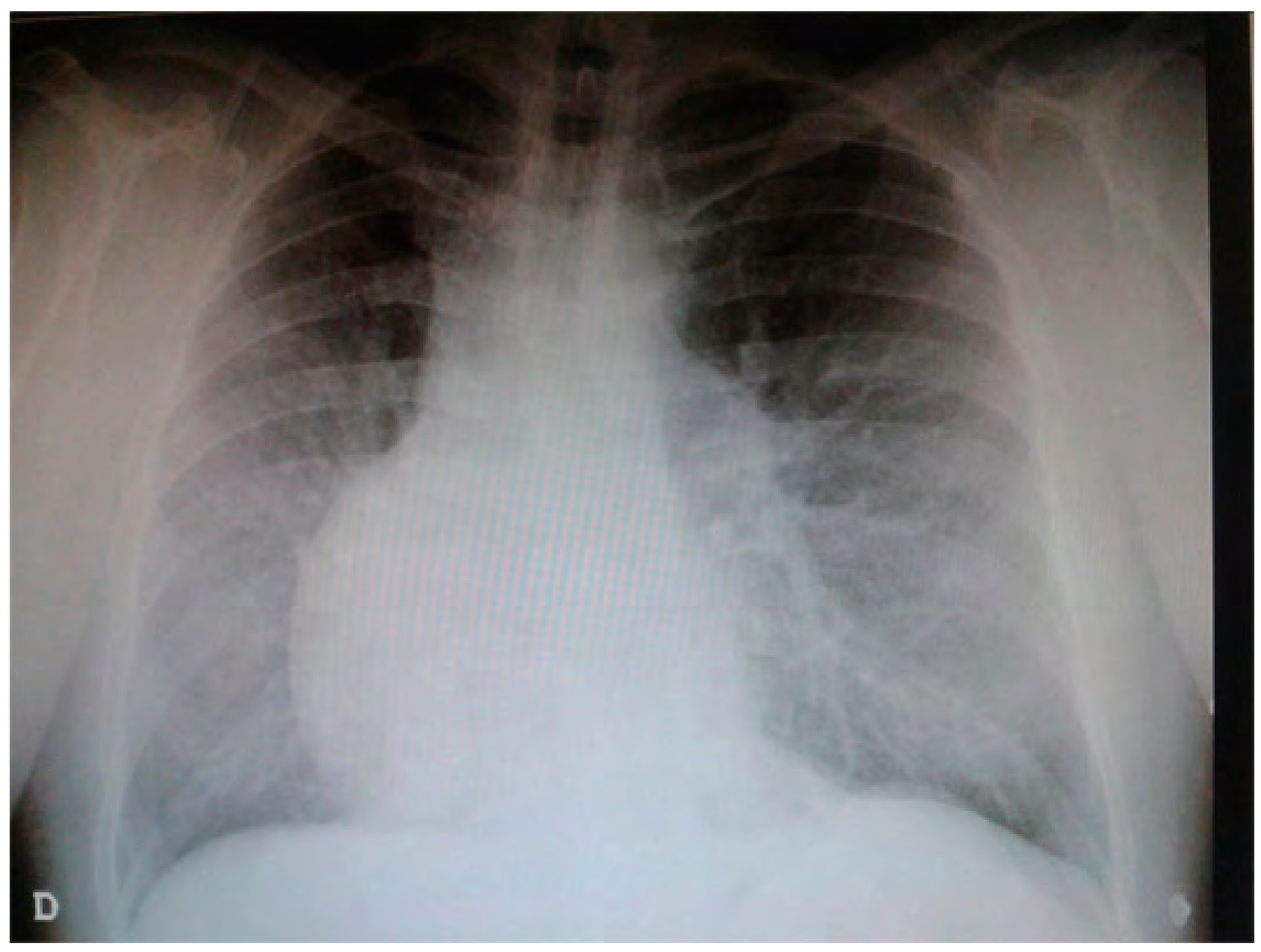

Figura 1. Radiografía de tórax del paciente con dextrocardia (no se aprecia el signo de la cimitarra). 


\section{DISCUSIÓN}

El síndrome de la cimitarra es una enfermedad congénita que implica una asociación de anomalías cardiopulmonares. Existe un drenaje venoso pulmonar anómalo hacía la vena cava superior o al ventrículo derecho. Se acompaña de hipoplasia de la arteria pulmonar derecha y consecuentemente del pulmón derecho, dextrocardia y un suministro anómalo desde la aorta al pulmón derecho, provocando un cortocircuito izquierda-derecha. La malformación cardiaca más frecuente es la comunicación interauricular (CIA) que se da en el $25 \%$ de los casos ${ }^{4}$.

La incidencia de esta patología es desconocida porque aún existen muchos casos asintomáticos, pero se estima entre 1 y 3 por cada 100.000 nacidos vivos. Se detectan en cualquier etapa de la vida, aunque a mayor edad es más raro su diagnóstico. Es más común entre el sexo femenino y raramente se afecta el pulmón izquierdo. La causa se desconoce ${ }^{1,5,6}$.

La forma adulta suele cursar sin hipertensión portal (HTP) y con CIA pequeña, bien tolerado desde el punto de vista clínico y con un pronóstico bueno. Según la asociación de las distintas anomalías la sintomatología puede variar desde asintomático a sintomatología leve como infecciones respiratorias de repetición, normalmente del lóbulo pulmonar derecho inferior por el menor riesgo sanguíneo y la hemoptisis secundaria a la HTP. En la forma infantil la clínica comienza poco después del nacimiento con fallo pulmonar, cardiaco o ambos, existe HTP severa y un mal pronóstico con una alta mortali$\operatorname{dad}^{2,3}$.

Lo más significativo del síndrome de la cimitarra es el retorno venoso anómalo; esto se puede sospechar con la imagen radiográfica al visualizarse el signo de la cimitarra en el $70 \%$ de los casos ${ }^{4}$. Cuando no se detecta este signo suele ser por la dextrorrotación (imagen que también nos debe hacer sospechar) o porque el drenaje venoso deja de ser curvo y ancho. Este signo radiológico suele estar ausente en niños ${ }^{7}$. La mayoría de los casos se diagnostican por ecocardiografía, que identifica el trayecto del drenaje venoso, obstrucciones y lugar de desembocadura del vaso. La técnica transtorácica tiene limitaciones y por eso se recomienda completar con la transesofágica. La TAC y RNM nos identifican la vena pulmonar anómala y descartan otras asociaciones como el pulmón en herradura (el $80 \%$ de los niños con pulmón en herradura tiene el síndrome de la cimitarra). La angiografía y el cateterismo son las técnicas más útiles para confirmar el diagnóstico ${ }^{3,8}$.

La indicación o no de cirugía va a depender de los defectos del septo arterial, la HTP o la estenosis de la vena anómala. En niños se trata medicamente esperando el aumento de tamaño antes de corregir el defecto, pero si no hay respuesta al tratamiento o si existe HTP se aconseja adelantar la cirugía ${ }^{3}$.

Nos parece interesante repasar el síndrome de la cimitarra, patología no bien conocida probablemente por su baja incidencia. Ver una radiografía de tórax es muy común en la práctica diaria de atención primaria y reconocer la imagen radiológica de la cimitarra nos podría ser útil ante su hallazgo, más aun sabiendo que este síndrome es asintomático y que muchos diagnósticos se hacen simplemente por la radiografía.

\section{BIBLIOGRAFÍA}

1. Neill CA, Ferencz C, Sabiston DC, Sheldon H. The familial occurrence of hypoplastic right lung with systemic arterial supply and venous drainage "scimitar syndrome". Bull Johns Hopkins Hosp. 1996; 107: 1-21.

2. Fuentes Alonso M, Ferreira Moreno A, de Miguel Díez J, Aguado del Hoyo A, Guembe Urtiaga P, Hernández Fernández J. Síndrome de la cimitarra de diagnóstico en la edad adulta. Rev Patol Respir. 2009; 12 (Supl 1): 86-8.

3. Kahrom M, Kahrom H. Scimitar syndrome and evolution of managements. Pan Afr Med J. 2009; 3: 20.

4. Espinola-Zavaleta N, Játiva-Chávez S, Muñoz-Castellanos L, Zamora-González C. Aspectos clínicos y ecocardiográficos del síndrome de la cimitarra. Rev Esp Cardiol. 2006; 59 (3): 284-8.

5. Dupuis $C$, Charaf LA, Brevière GM, Abou P, Rémy-Jardin M, Helmius G. The "adult" form of the scimitar syndrome. Am J Cardiol. 1992; 70 (4): 502-7.

6. Rutledge JM, Hiatt PW, Wesley Vick G 3rd, Grifka RG. A sword for the left hand: an unusual case of left-sided scimitar syndrome. Pediatr Cardiol. 2001; 22 (4): 350-2.

7. Canter CE, Martin TC, Spray TL, Weldon CS, Strauss AW. Scimitar syndrome in childhood. Am J Cardiol. 1986; 58 (7): 652-4.

8. Dupuis C, Vaksmann G, Reéy-Jardin M, Francart C. Horseshoe lung and scimitar syndrome in an asymptomatic child. Eur Heart J. 1994; 15 (7): 1008-9. 